\title{
The Intricate Determination of Magnetic Anisotropy in Quasi-octahedral Vanadium(III): An HF-EPR and Magnetic Study
}

\author{
Kátia Cristina Molgero Westrup ${ }^{1}$ (D) - Francielli Sousa Santana ${ }^{1}$ (D) . \\ David Lewis Hughes ${ }^{2}$ (D) Giovana Gioppo Nunes ${ }^{1}$ (D) \\ Jaísa Fernandes Soares ${ }^{1}$ (D) . Anne-Laure Barra ${ }^{3} \cdot$ Roberta Sessoli $^{4,5}$ (D) \\ Lorenzo Sorace ${ }^{4}$ (D)
}

Received: 30 June 2020 / Revised: 2 September 2020 / Published online: 22 September 2020

(c) The Author(s) 2020

\begin{abstract}
We report here the synthesis and a preliminary characterization of the tetranuclear complex of formula $\left[\mathrm{Ga}_{3} \mathrm{~V}\left(\mathrm{~L}^{\mathrm{Et}}\right)_{2}(\mathrm{dpm})_{6}\right], \mathbf{G a}_{3} \mathbf{V E t}$, in which $\boldsymbol{H}_{3} \boldsymbol{L}^{\boldsymbol{E} \boldsymbol{t}}=2$-Ethyl-2(hydroxymethyl)-propane-1,3-diol and $\mathrm{Hdpm}=$ dipivaloylmethane, containing a single paramagnetic vanadium(III) center, from a structural, magnetic, and spectroscopic point of view. Structural characterization by X-ray diffraction evidenced that this derivative is isostructural with the star-shaped Single-Molecule Magnet $\left[\mathrm{Fe}_{3} \mathrm{~V}\left(\mathrm{~L}^{\mathrm{Et}}\right)_{2}(\mathrm{dpm})_{6}\right], \mathbf{F e}_{\mathbf{3}} \mathbf{V E t}$, and can, thus, be considered a model to analyze the magnetic anisotropy of the vanadium(III) ion in that system. The observed results confirm the complexity in obtaining a rationalization of the magnetic behavior of this metal ion, with magnetization data and High Field Electron Paramagnetic Resonance (HF-EPR) spectroscopy providing apparently conflicting results. Indeed, the former were rationalized assuming a rhombic distortion of the ligand field and a dominant easy-axis type anisotropy (equivalent to $D \approx-14.1 \mathrm{~cm}^{-1}, E \approx 1.2 \mathrm{~cm}^{-1}$ ), while a simple axial Spin Hamiltonian approach could explain HF-EPR data $(|D| \approx$ $\left.6.98 \mathrm{~cm}^{-1}\right)$.
\end{abstract}

Electronic supplementary material The online version of this article (https://doi.org/10.1007/s0072 3-020-01265-3) contains supplementary material, which is available to authorized users.

\section{Lorenzo Sorace}

lorenzo.sorace@unifi.it

1 Departamento de Química, Universidade Federal do Paraná, Curitiba, PR 81530-900, Brazil

2 School of Chemistry, University of East Anglia, Norwich NR4 7TJ, UK

3 Laboratoire National des Champs Magnétiques Intenses-CNRS, Université Grenoble-Alpes, 38042 Grenoble Cedex 9, France

4 Department of Chemistry "U. Schiff” and Udr INSTM, University of Florence, Via della Lastruccia 3, 50019 Sesto Fiorentino, Italy

5 ICCOM-CNR, Via Madonna del Piano 10, 50019 Sesto Fiorentino, FI, Italy 


\section{Introduction}

The discovery in the early 90s of Single-Molecule Magnet (SMM) behavior, i.e., magnetic bistability at the molecular level in polynuclear transition metal complexes [1], sparked intense research in the field of molecular magnetism, aiming at the possible use of these systems as magnetic memory units [2]. The cause of the slow relaxation of the magnetization in these systems was soon attributed to the existence of an anisotropy barrier that amounts to the total energy spanned by the ground spin state, S, of the complexes [3]. For a system with an integer spin state and an easy axis of magnetization, this is equal to $|D| \mathrm{S}^{2}$, where $D$ is the parameter describing the zero-field splitting (i.e., magnetic anisotropy) of the $S$ multiplet $(D<0)$. When thermal energy is much lower than the height of the barrier, a system previously magnetized will retain its magnetization; on the contrary, on increasing temperature, thermal energy becomes large enough to allow fast relaxation of the magnetization, leading to a loss of the information stored in it. This prompted many groups to find ways to improve the magnetic anisotropy barrier rationally [4, 5]. This is, however, by no means a simple task: in polynuclear clusters, it requires careful engineering of the anisotropic features of the constituent ions, the relative orientation of their magnetic anisotropy axis, and the exchange coupling among the centers [6]. In this context, one of the most accurately studied families is that of tetranuclear star-shaped complexes generally referred to as $\mathbf{F e}_{\mathbf{3}} \mathbf{M}^{\prime}\left(\mathbf{M}^{\prime}=\mathrm{Fe}, \mathrm{Cr}\right.$, V, see Scheme 1) [7-10], which also proved very successful in nanostructuration processes [11-13].

For this family, some of us showed in the past that changing the central metal $\mathrm{M}^{\prime}$ allows playing with both factors affecting the magnetic anisotropy barrier height. By changing $\mathrm{M}^{\prime}$ from iron(III) to chromium(III) and vanadium(III), the global spin state increased from $S=5$ to $S=6$ to $S=13 / 2[8,9]$. At the same time, this modification affected the anisotropy parameter $D$, as a consequence of both the different singleion contribution of the central site and the different ratio between single-ion and

Scheme 1 General structure of star-shaped $\mathbf{F e}_{\mathbf{3}} \mathbf{M}^{\prime}$ SMMs, depicting the tripodal alkoxide ligands above and below the plane defined by the metal ions. The wireframe colors are gray for carbon and red for oxygen atoms. The alkyl or aryl groups bound to the bridgehead carbon in each chelating alkoxide (Et, $\mathrm{Me}, \mathrm{Ph}$, and $\mathrm{Bu}^{\mathrm{t}}$ in different literature reports) are not shown (colour figure online)

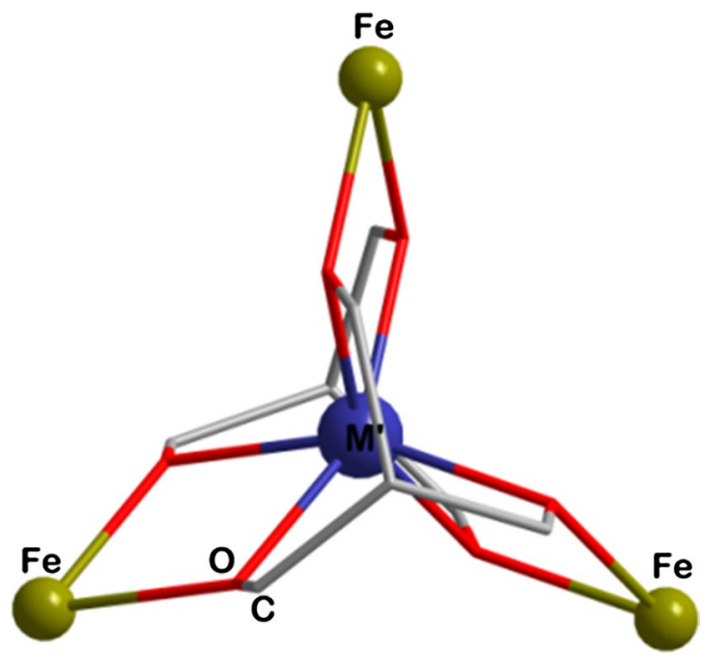


global spin states. While the anisotropy in $\mathbf{F e}_{3} \mathbf{C r}$ and $\mathbf{F e}_{3} \mathbf{F e}$ could be rationalized entirely on the basis of single-ion and dipolar anisotropy as derived by the study of iron(III)- and chromium(III)-doped isostructural $\mathbf{G a}_{4}$ diamagnetic clusters [8], the situation remained unclear for $\mathbf{F e}_{3} \mathbf{V}$. At that time, $\mathbf{G a}_{3} \mathbf{V}$ was not available, and we resorted to simple Ligand Field arguments to obtain a rough estimate of the singleion anisotropy of vanadium(III), which is an orbitally degenerate ion in octahedral symmetry $\left({ }^{3} T_{1 g}\right)$. As deeply discussed in a series of paper by the late Tregenna-Piggott, trigonal distortion may provide either an orbitally non-degenerate ground state, with hard-axis anisotropy, or an orbitally degenerate ground state, whose anisotropic properties cannot be directly mapped onto a Spin Hamiltonian in the absence of further rhombic distortions [14-16]. This complicates the analysis of the origin of the anisotropy in exchange coupled systems containing vanadium(III) [17] and, in the case of $\mathbf{F e}_{3} \mathbf{V}$, a non-negligible contribution of anisotropic exchange was postulated [9]. The isostructural $\mathbf{G a}_{3} \mathbf{V}$ derivative has now been obtained, and we report here some preliminary results concerning its structural, High Field Electron Paramagnetic Resonance (HF-EPR), and magnetic properties, which evidence the complexity of the rationalization of this metal ion and suggest that our previous estimate might be wrong.

\section{Materials and Methods}

\subsection{General}

Syntheses were carried out under $\mathrm{N}_{2}$ (99.999\%, Praxair) with the use of Schlenk and glove-box techniques. Solvents (Aldrich and Vetec) were dried by standard procedures [18] and distilled under $\mathrm{N}_{2}$ before use. Anhydrous vanadium(III) chloride, anhydrous gallium(III) chloride, dipivaloylmethane (Hdpm), and n-butyllithium (2.5 mol L-1 solution in hexanes) were purchased from Aldrich and used without additional purification. 2-Ethyl-2-(hydroxymethyl)-propane-1,3-diol $\left(\mathbf{H}_{3} \mathbf{L}^{\mathrm{Et}}\right.$, Aldrich) was recrystallized from a 3:1 toluene/tetrahydrofuran (thf) with nearly quantitative yield, as described previously [19]. $\left[\mathrm{VCl}_{3}(\mathrm{thf})_{3}\right]$ [20] and $\left[\mathrm{Ga}_{2}(\mathrm{OMe})_{2}(\mathrm{dpm})_{4}\right][8]$ were prepared by the published methods.

Elemental analyses were carried out by Medac Laboratories Ltd. (Chobham, Surrey, UK). The equipment employed was a Thermal Scientific Flash EA 1112 Series Elemental Analyser (C and H contents) and a Varian Vista MPX ICP-OES spectrometer for Gallium and Vanadium contents. Infrared spectroscopy data presented as Supplementary Material $\left(400-4000 \mathrm{~cm}^{-1}\right.$, Figure S1) were recorded from $\mathrm{KBr}$ pellets on BIORAD FTS3500GX equipment with a spectral resolution of $2 \mathrm{~cm}^{-1}$.

\subsection{Synthesis of $\left[\mathrm{Ga}_{3} \mathrm{~V}\left(\mathrm{~L}^{\mathrm{Et}}\right)_{2}(\mathrm{dpm})_{6}\right]\left(\mathrm{Ga}_{3} \mathrm{VEt}\right)$}

The preparation was carried out by the procedure previously employed for $\mathrm{Fe}_{3} \mathbf{C r E t}$ [19] and $\mathbf{F e}_{3} \mathrm{VEt}$ [9]. A solution of $\mathrm{H}_{3} \mathrm{~L}^{\mathrm{Et}}$ (230 mg, $\left.1.51 \mathrm{mmol}\right)$ in cold thf $\left(30 \mathrm{~mL}, 0{ }^{\circ} \mathrm{C}\right)$ received the dropwise addition of n-butyllithium $(4.50 \mathrm{mmol})$. 
The resulting white suspension was kept stirring at $0{ }^{\circ} \mathrm{C}$ for $5 \mathrm{~h}$ and was then filtered to give a quantitative yield of the tripodal lithium alkoxide $\mathrm{Li}_{3} \mathrm{~L}^{\mathrm{Et}}$. The white solid was dried under vacuum and immediately used as follows. Solid $\left[\mathrm{VCl}_{3} \text { (thf) }\right)_{3}$ (280 mg, $\left.0.749 \mathrm{mmol}\right)$ was slowly extracted for $48 \mathrm{~h}$ into a boiling suspension of $\mathrm{Li}_{3} \mathrm{~L}^{\mathrm{Et}}(221 \mathrm{mg}, 1.45 \mathrm{mmol})$ in 1,2-dimethoxyethane (glyme, $120 \mathrm{~mL}$ ). This produced a light-brown suspension that was added at room temperature to a white suspension of $\left[\mathrm{Ga}_{2}(\mathrm{OMe})_{2}(\mathrm{dpm})_{4}\right](1.04 \mathrm{~g}, 1.11 \mathrm{mmol})$ in glyme $(50 \mathrm{~mL})$. The resulting mixture was heated under reflux for $5 \mathrm{~h}$, cooled down to room temperature, and then filtered, producing a greenish-brown solid mixed with a white contaminant (possibly $\mathrm{LiCl}$ and $\mathrm{LiOMe}$ ). This solid was extracted with thf, and the light yellow extract was submitted to vapor diffusion of glyme over 4 weeks. From the crystallization chamber, brownishgreen crystals $\left(\mathbf{G a}_{3} \mathbf{V E t}-1\right)$ were isolated by filtration and dried under vacuum. Total yield $0.23 \mathrm{~g}$ ( $\mathrm{ca}$. 19\%). Elemental analysis contents calculated (\%) for $\mathrm{C}_{78} \mathrm{H}_{136} \mathrm{O}_{18} \mathrm{VGa}_{3}$ : C 57.76, $\mathrm{H}$ 8.45, Ga 12.90, V 3.14; found (\%) for $\mathbf{G a}_{3} \mathbf{V E t}-1$ : C 57.45, $\mathrm{H}$ 8.39, Ga 12.92, V 2.95. $\mathrm{Ga}_{3} \mathrm{~V} / \mathrm{Ga}_{4}$ ratio $(\% \mathrm{~m} / \mathrm{m}): 95.2 / 4.8$.

An analogous procedure with the reaction time of $72 \mathrm{~h}$ in the extraction step gave crystals labeled as $\mathbf{G a}_{3} \mathbf{V E t}-2$, and an increase in total yield: $0.34 \mathrm{~g}$ (ca. 28\%). Elemental analysis found (\%) for Ga $\mathbf{G}_{3}$ VEt-2: C 57.48, H 8.49, Ga 13.09, $\mathrm{V}$ 2.92. $\mathrm{Ga}_{3} \mathrm{~V}: \mathrm{Ga}_{4}$ ratio $(\% \mathrm{~m} / \mathrm{m}):$ 93.5: 6.5 .

Samples of $\mathbf{G a}_{3} \mathbf{V E t}$ can be handled in air in the solid state for several days without detectable change. Their structural, spectroscopic, and magnetic characterization was performed in air.

\subsection{Crystal Structure Analysis of $\mathrm{Ga}_{3} \mathrm{VEt}$}

Crystal data: $\mathrm{C}_{78} \mathrm{H}_{136} \mathrm{Ga}_{3} \mathrm{O}_{18} \mathrm{~V}, M=1621.96$. Trigonal, space group $R-3 c: h$ (no. 167:h), $a=b=16.4261(4) \AA, \quad c=57.838(2) \AA, \quad \alpha=\beta=90^{\circ}, \quad \gamma=120^{\circ}$, $V=13,514.9(9) \AA^{3} . Z=6, D_{\text {calc }}=1.196 \mathrm{~g} \mathrm{~cm}^{-3}, F(000)=5184, T=273(2) \mathrm{K}$, $\lambda(\mathrm{Mo}-K \alpha)=0.71073 \AA$ A.

Crystals are greenish-brown rhombohedra. From a sample under oil, one, $c a$ $0.46 \times 0.40 \times 0.23 \mathrm{~mm}$, was mounted on a micro-mount and fixed in the cool nitrogen stream on a Bruker D8 Venture diffractometer equipped with a Photon $100 \mathrm{CMOS}$ detector, Mo- $K \alpha$ radiation, and graphite monochromator. Intensity data were measured at 273(2) $\mathrm{K}$ by thin-slice $\omega$ - and $\varphi$-scans. The total number of reflections recorded, to $\theta_{\max }=25^{\circ}$, was 146490 of which 2651 were unique $[R($ int $)=0.056] ; 2186$ were 'observed' with $I>2 \sigma_{I}$.

Data were processed using the Bruker APEX2 software [21]. The structure was determined by direct methods in the SHELXS program [22] and refined by full-matrix least-squares methods, on $\mathrm{F}^{2}$ s, in SHELXL [23]. Scattering factors for neutral atoms were taken from the literature [24]. All computer programs used in these analyses were run through WinGX $[25,26]$ at the Universidade Federal do Paraná and the University of East Anglia. Crystal data, data collection, and general structure refinement data are presented in Table S1. 


\subsection{Notes on the Structure}

Figure S2 presents the first coordination sphere of the central (vanadium) ion, while Figures S3 and S4 show different views of the tetranuclear structure, with the atom numbering scheme. The asymmetric unit of $\left[\mathrm{V}\left\{\left(\mathrm{OCH}_{2}\right)_{3} \mathrm{CEt}\right\}_{2}\left\{\mathrm{Ga}(\mathrm{dpm})_{2}\right\}_{3}\right]$ (Figure S5) contains two different metal centers in special positions: a $\mathrm{Ga}^{\mathrm{III}}$ ion that lies on a twofold axis and a $\mathrm{V}^{\mathrm{III}}$ ion lying on several symmetry elements: one threefold axis (parallel to $c$ ), three twofold axes (one parallel to $b$, the second one parallel to $a$, and the third bisecting the angle between the first two), and three glide planes. The asymmetric unit (Figure S5) also contains a complete $\beta$-diketonate $\left(\mathrm{dpm}^{-}\right)$ligand coordinated to $\mathrm{Ga}^{\mathrm{III}}$, and one-third of a tripodal alkoxide ligand (including one of the disordered orientations of its ethyl group) bridging the vanadium and gallium ions through the $\mathrm{O} 3$ donor atom.

A high degree of disorder affects the tert-butyl groups of the $\beta$-diketonate ligands. In the asymmetric unit, the disorder involving the carbon atoms attached to $\mathrm{C} 4(\mathrm{C} 1-\mathrm{C} 3)$ was modeled with two orientations and occupancy factors of 0.56 and 0.44. In the minor component, the $\mathrm{C} 100, \mathrm{C} 200$, and $\mathrm{C} 300$ atoms were refined isotropically; these were the only non-hydrogen atoms refined in this way. Disorder was also evident for $\mathrm{C} 15$ in the ethyl group of the tripodal alkoxide; this atom was refined with an occupancy factor of 0.33 as a consequence of the threefold symmetry axis passing through $\mathrm{C} 14$ and $\mathrm{C} 13$. The distance restraint DFIX was applied to all disordered atoms mentioned above: C1, C2, C3, C15, C100, C200, and C300. All $\mathrm{B}$ alerts in the CheckCIF file refer to these disordered portions of the asymmetric unit.

Hydrogen atoms were included in idealized positions and were refined isotropically; their $U_{\text {iso }}$ values were set to ride on the $U_{\text {eq }}$ values of the parent carbon atoms.

No hydrogen bond or other intra/intermolecular interaction of considerable strength was found in the structure.

Replacement of the central vanadium atom by gallium was investigated, but no improvement was seen; the best refinement was reached with a $\mathbf{G a}_{\mathbf{3}} \mathbf{V}$ system.

\subsection{Magnetic Characterization}

The magnetization of the sample was measured using an MPMS SQUID magnetometer (Quantum Design, Inc.). Microcrystalline powders were pressed in a pellet to avoid preferential orientation of the crystallites due to magnetic torque. Raw data were corrected for the sample holder contribution, measured in the same field and temperature range, and for the intrinsic diamagnetism of the sample, estimated using Pascal's constants [27]. Fits and simulations of magnetic data were obtained using PHI [28].

\subsection{High Field Electron Paramagnetic Resonance (HF-EPR)}

HF-EPR measurements were performed on a home-built multi-frequency spectrometer operating in a double-pass configuration. A $110 \mathrm{GHz}$ frequency source (Virginia 
Diodes Inc.) alone or with multipliers up to the third harmonic), as well as 95 and $115 \mathrm{GHz}$ Gunn oscillators (Radiometer Physics $\mathrm{GmbH}$ ) together with multipliers up to the fourth harmonic were used. The main magnetic field was supplied by a $16 \mathrm{~T}$ superconducting magnet associated with a Variable Temperature Insert (Cryogenic). A hot electron InSb bolometer (QMC Instruments) was used to measure the light transmitted through the sample. The spectrometer is designed to operate in the perpendicular mode, which means that the oscillating microwave field $\boldsymbol{B}_{\boldsymbol{m} w}$ is perpendicular to the static $\boldsymbol{B}_{d c}$ and the selection rule for the transition is thus $\Delta M_{\mathrm{S}}= \pm 1$. The measurements were done on the microcrystalline powder sample pressed into a pellet in order to limit torqueing effects (actually, the same pellet of $\mathrm{ca} .12 \mathrm{mg}$ which was used for SQUID measurements), in the 190-460 GHz frequency range at 5 and 15 K. Spectral simulation and analysis were performed using EasySpin [29].

\section{Results}

\subsection{Synthesis and Structure}

Previous works described several propeller-like complexes with the general formula $\left[\mathrm{M}_{4}\left(\mathrm{~L}^{\mathrm{R}}\right)_{2}(\mathrm{dk})_{6}\right]$, in which Hdk is a $\beta$-diketone and $\mathrm{H}_{3} \mathrm{~L}^{\mathrm{R}}$ is a tripodal alcohol, containing different alkyl or aryl $(\mathrm{R})$ groups in the tripodal ligand $[8,10,19,30]$. Synthetically speaking, the nature of the R group influences the solubility of the intermediate species in the three-step procedure (see below), and of the final complex, modifying the reaction yield. The choice of ethyl as the $\mathrm{R}$ group in the tripodal alkoxide was made in this case to allow comparison with the heterometallic $\mathbf{M}_{\mathbf{3}} \mathbf{M}^{\prime}$ species available in the literature, and in particular with $\mathbf{F e}_{\mathbf{3}} \mathbf{V}$.

The complex $\left[\mathrm{Ga}_{3} \mathrm{~V}\left(\mathrm{~L}^{\mathrm{Et}}\right)_{2}(\mathrm{dpm})_{6}\right] \quad\left(\mathbf{G a}_{3} \mathbf{V E t}\right)$, in which $\mathrm{H}_{3} \mathrm{~L}^{\mathrm{Et}}=2$-ethyl-2(hydroxymethyl)-propane-1,3-diol and $\mathrm{Hdpm}=$ dipivaloylmethane, was prepared by the three-step route developed earlier for the isostructural $\mathbf{F e}_{\mathbf{3}} \mathbf{M}^{\prime} \mathbf{E t}$ compounds in which $\mathrm{M}^{\prime}=\mathrm{Cr}^{\mathrm{III}}$ and $\mathrm{V}^{\mathrm{III}}[9,19]$ :

$$
\begin{aligned}
& H_{3} L^{E t}+3 B u^{n} L i \rightarrow L i_{3} L^{E t}+3 B u^{n} H(\text { step } 1) \\
& {\left[\mathrm{VCl}_{3}(\mathrm{thf})_{3}\right]+2 \mathrm{Li}_{3} L^{E t} \rightarrow \mathrm{Li}_{3} V\left(L^{E t}\right)_{2}+3 \mathrm{LiCl}+3 \operatorname{thf}(\mathrm{step} 2)} \\
& \mathrm{Li}_{3} V\left(\mathrm{~L}^{E t}\right)_{2}+1.5\left[\mathrm{Ga}_{2}(\mathrm{OMe})_{2}(\mathrm{dpm})_{4}\right] \rightarrow\left[\mathrm{Ga} \mathrm{a}_{3}\left(\mathrm{~L}^{\mathrm{Et}}\right)_{2}(\mathrm{dpm})_{6}\right]+3 \mathrm{LiOMe} \text {. (step3) }
\end{aligned}
$$

In agreement with our previous findings [9], step 2 was confirmed to be crucial to the success of the synthesis and isolation of a chemically pure heterometallic $\mathrm{M}_{3} \mathrm{M}^{\prime}$ species. This step leads to the formation of the central portion ("core", formulated as $\mathrm{V}\left(\mathrm{L}^{\mathrm{Et}}\right)_{2}^{3-}$, Fig. 1, left) of the star-shaped compound, around which the propeller "blades", $\mathrm{Ga}(\mathrm{dpm})_{2}{ }^{+}$, assemble themselves in the last reaction step. We found that, for each tetra(hetero)nuclear compound, different reaction conditions had to be adjusted to maximize the global reaction yield, depending on the chemical nature of the central ion. As far as the preparation of $\mathbf{G a}_{\mathbf{3}} \mathbf{V E t}$ is concerned, longer and slower 

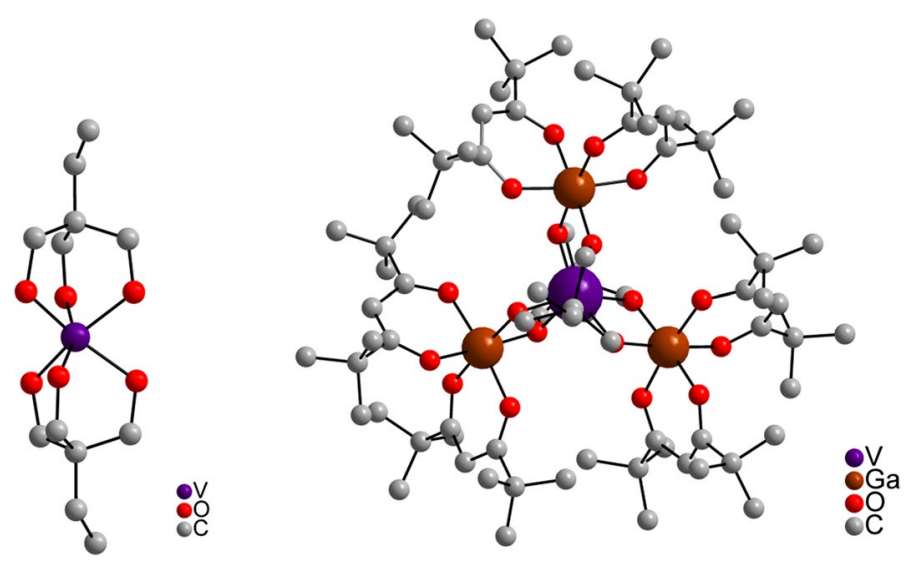

Fig. 1 Left: representation [31] of the $\mathrm{V}\left(\mathrm{L}^{\mathrm{Et}}\right)_{2}^{3-}$ "core" in $\mathbf{G a} \mathbf{a}_{\mathbf{3}} \mathbf{V E t}$. Only one of the three equivalent, disordered orientations of the ethyl group about the threefold symmetry axis is shown. Right: general view [31] of the $\left[\mathrm{Ga}_{3} \mathrm{~V}\left(\mathrm{~L}^{\mathrm{Et}}\right)_{2}(\mathrm{dpm})_{2}\right]\left(\mathbf{G a}_{3} \mathbf{V E t}\right)$ molecular structure down the threefold symmetry axis. The disordered orientations of the ethyl group in the tripodal alkoxides and of the tert-butyl groups on the $\beta$-diketonate $\left(\mathrm{dpm}^{-}\right)$ligands were omitted for clarity. ORTEP-3 [26] diagrams, with the atom numbering scheme, are presented in Figures S3 and S4

extraction of the vanadium(III) starting material into the boiling lithium alkoxide suspension in step 2 gives the best yields of the product, as described in the Experimental section.

The addition of the gallium(III) dimer $\left[\mathrm{Ga}_{2}(\mathrm{OMe})_{2}(\mathrm{dpm})_{4}\right]$ to the suspension of the "core" in dimethoxyethane (step 3), followed by heating under reflux, gives a greenish-(light)brown solution from which microcrystals of $\mathbf{G a}_{\mathbf{3}} \mathbf{V E t}$ and a white powder, probably a mixture of $\mathrm{LiCl}$ and $\mathrm{LiOMe}$, are isolated by filtration. This impure product is then recrystallized by vapor diffusion of dimethoxyethane into a thf solution, producing beautiful greenish-(light)brown rhombohedra.

Despite the well-formed appearance of the crystals, they produce weak X-ray diffraction patterns, even at low temperature, because of static and dynamic disorder in the ethyl group of the tripodal alkoxides and in the tert-butyl groups of the $\beta$-diketonate $\left(\mathrm{dpm}^{-}\right)$ligands. This disorder is common to several members of the star-shaped $\mathbf{M}_{4}$ family reported to date [8, 30, 32], and, in the case of $\mathbf{G a}_{\mathbf{3}} \mathbf{V E t}$, it hampered the obtainment of an accurate X-ray structural description of the molecule. After many attempts involving distinct synthesis and recrystallization conditions, we have been able to confirm that the tetranuclear molecules found in the crystals of $\mathbf{G a}_{\mathbf{3}} \mathbf{V E t}$ (Figs. 1 right, S3 and S4) are isostructural, as expected, with the 'star' structures reported for $\mathbf{F e}_{\mathbf{3}} \mathbf{C r E t}$ [19] and $\mathbf{F e}_{\mathbf{3}} \mathbf{V E t}$ [9] in the same trigonal space group $R-3 c: h$, and that the bond distances and angles in all three complexes are similar (Tables 1, S1-S3). The lower quality of the X-ray diffraction for $\mathbf{G a}_{\mathbf{3}} \mathbf{V E t}$ compared to $\mathbf{F e}_{3} \mathbf{C r E t}$ [19] and $\mathrm{Fe}_{3} \mathbf{V E t}$ [9] provides a higher incertitude in the geometrical parameters. However, it allows us to establish the shape and symmetry of the molecule.

Further evidence in favor of the composition of the crystalline product came from elemental analysis (see Experimental) and spectroscopic data, as described below 
Table 1 Selected structural data for propeller-like complexes

\begin{tabular}{|c|c|c|c|}
\hline $\begin{array}{l}\text { Average bond lengths and non-bonding } \\
\text { distances }(\AA)\end{array}$ & $\begin{array}{l}\mathrm{Fe}_{3} \mathrm{M}^{\prime} \mathrm{Et} \\
(293 \mathrm{~K}) \\
\left(\mathrm{M}^{\prime}=\mathrm{V}, \mathrm{Fe}\right)[9]\end{array}$ & $\begin{array}{l}\mathrm{Fe}_{3} \mathrm{CrEt} \\
(293 \mathrm{~K})[19]\end{array}$ & $\begin{array}{l}\mathrm{Ga}_{3} \mathrm{VEt} \\
(273 \mathrm{~K})\end{array}$ \\
\hline $\mathrm{M}_{\text {central }}-\mathrm{O}_{\text {tripodal }}$ & $1.977(2)$ & $1.949(3)$ & $1.999(8)$ \\
\hline $\mathrm{M}_{\text {peripheral }}-\mathrm{O}_{\text {tripodal }}$ & $1.963(2)$ & $1.963(3)$ & $1.922(7)$ \\
\hline $\mathrm{M}_{\text {peripheral }}-\mathrm{O}_{\text {diketonate (average) }}$ & $1.993(2)$ & $1.991(4)$ & $1.974(9)$ \\
\hline $\mathrm{M}_{\text {central }} \cdots \mathrm{C}_{\text {central }}{ }^{\mathrm{a}}$ & 3.029 & 2.986 & 3.004 \\
\hline$M_{\text {central }} \cdots M_{\text {peripheral }}$ & 3.075 & 3.023 & 3.036 \\
\hline $\mathrm{M}_{\text {peripheral }} \cdots \mathrm{M}_{\text {peripheral }}$ & 5.326 & 5.237 & 5.258 \\
\hline $\begin{array}{l}\text { Effective ionic radius (central ion) }(\AA) \text { [ } 40 \text {, } \\
41]\end{array}$ & $0.640 / 0.645^{\mathrm{b}}$ & 0.615 & 0.640 \\
\hline Effective ionic radius (peripheral ion) $(\AA)$ & 0.645 & 0.645 & 0.620 \\
\hline Helical pitch angle ${ }^{\mathrm{c}}\left(^{\circ}\right)$ & 69.8 & 63.4 & 66.0 \\
\hline Bond precision $\mathrm{C}-\mathrm{C}^{\mathrm{d}}(\AA)$ & 0.0091 & 0.0117 & 0.0248 \\
\hline
\end{tabular}

Temperatures of data collection are given in parentheses

Bond angles for the three complexes are compiled in Table S2, while Table S3 presents the complete listing of bond distances and angles for $\mathbf{G a}_{\mathbf{3}} \mathbf{V E t}$

${ }^{\mathrm{a}} \mathrm{C}_{\text {central }}=$ central (bridgehead) carbon atom in the tripodal alkoxide ligand, $\left[\mathrm{R} \underline{\mathbf{C}}\left(\mathrm{CH}_{2} \mathrm{O}\right)_{3}\right]^{3-}$

${ }^{\mathrm{b}}$ For V VII and high-spin Fe ${ }^{\mathrm{III}}$, respectively. Crystals contained $\mathbf{F e}_{\mathbf{3}} \mathbf{V E t}$ and $\mathbf{F e}_{\mathbf{4}} \mathbf{E t}$ in $46: 54$ wt\% proportion [9]

${ }^{\mathrm{c}}$ Dihedral angle between the planes defined by the $\mathrm{M}_{\text {central }}(\mathrm{O})_{2} \mathrm{M}_{\text {peripheral }}$ units and the four metal ions ${ }^{\mathrm{d}}$ From CheckCIF (https://checkcif.iucr.org/)

and in the Supplementary Material. Metal analysis results indicate $c a .95 \%$ chemically pure samples as far as the $\mathbf{G a}_{\mathbf{3}} \mathbf{V}$ formulation is concerned. This is particularly relevant considering that the modular synthesis described in steps 1-3 competes with the self-assembly of homometallic species (in this case, $\mathbf{G a}_{4} \mathbf{E t}$ ), as discussed earlier $[8,9,19]$. This success in the desired synthesis of the heterometallic $\mathbf{G a}_{3} \mathbf{V}$ entity probably relates both to the chelate effect stabilizing the vanadium "core" assembled in step 2, and the similar labilities of vanadium(III) and gallium(III) towards ligand substitution reactions [33-37]; as a consequence, none of the homometallic $\mathrm{Ga}_{4}$ or $\mathrm{V}_{4}$ structures is particularly favored when compared to the heterometallic species. The loss of vanadium(III) from the "core" to give $c a .5 \% \mathbf{G a}_{4}$ (see elemental analysis results) could come from the lower affinity of the softer $\mathrm{V}^{\mathrm{III}}$ ion (as compared to $\mathrm{Ga}^{\mathrm{III}}$ ) towards the hard oxygen-donor atoms of the tripodal alkoxide $[38,39]$.

Figures 1 right, S3, and S4 confirm the star-shaped, propeller-like molecular structure of a new component of the $\left[\mathrm{M}_{3} \mathrm{M}^{\prime}\left(\mathrm{L}^{\mathrm{Et}}\right)_{2}(\mathrm{dpm})_{6}\right]$ series. In addition, data presented in Tables 1, S2 and S3 give evidence in favor of the desired $\mathrm{M}=\mathrm{Ga}^{\mathrm{III}}$ and $\mathrm{M}^{\prime}=\mathrm{V}^{\mathrm{III}}$ identities for the peripheral and central metal ions respectively, and reassert that the synthetic route summarized in steps 1-3 is an efficient pathway to pure heterometallic $\mathrm{M}_{3} \mathrm{M}^{\prime}$ species.

In Table 1 (last column), all $\mathrm{M}_{\text {central }}$ and $\mathrm{M}_{\text {peripheral }}$ bond distances to the $O$-donor atoms of the tripodal alkoxide and $\beta$-diketonate ligands support a larger 
ion ( $\mathrm{V}^{\mathrm{III}}$; effective atomic radius $0.640 \AA$ ) at the center of the molecular "core", and a smaller cation $\left(\mathrm{Ga}^{\mathrm{III}}\right.$, effective atomic radius $\left.0.620 \AA\right)$ in the peripheral positions. This size ordering is the inverse of that observed for the $\mathrm{Cr} \mathrm{rII}^{\mathrm{II}} / \mathrm{Fe}^{\mathrm{III}}$ pairs in $\mathbf{F e}_{3} \mathbf{C r E t}$ [19], which is also in agreement with the ionic radii magnitudes. During structure refinement, attempts to replace the central vanadium(III) ion in $\mathbf{G a}_{3} \mathbf{V E t}$ with gallium(III) to check for the possible formation of $\mathbf{G a}_{\mathbf{4}} \mathbf{E t}$ led to higher R-factors and significant change (increase or decrease) in the anisotropic thermal parameters, indicating the worsening of the structural model. This result supports the presence of vanadium in the molecular "core".

Taking the small difference in temperature of data collection into consideration, data in Tables 1 and S2, together with unit cell volumes [13730.5(8) $\AA^{3}$ for $\mathbf{F e}_{3} \mathbf{C r E t}$; 13740.4(5) $\AA^{3}$ for $\mathbf{F e}_{3} \mathbf{V E t}[9,19]$ and 13514.9(9) $\AA^{3}$ for $\mathbf{G a}_{3} \mathbf{V E t}$, indicate no large differences in the molecular shape or global size on changing the nature of the central/peripheral metal ions across the fourth period of the periodic classification. These changes are well known to be crucial, however, for the easyor hard-axis nature of the magnetic anisotropy in the entire molecular assembly, as well as to the height of the magnetization reversal barrier [8,30].

An interesting observation can be made at a more localized level: all bond angles involving the peripheral ions are significantly closer to the octahedral values in $\mathbf{G a}_{3} \mathbf{V E t}$ than in $\mathbf{F e}_{3} \mathbf{C r E t}$ or $\mathbf{F e}_{3} \mathbf{V E t}$ (Table S2), leading to a more regular geometry for the peripheral units. This distinguishes $\mathbf{G a}_{\mathbf{3}} \mathbf{V E t}$ from the other two systems in which iron(III) ions are bound to the diketonate ligands. Additionally, the observation of the bond angles about the central atom in Table S2 shows an interesting similarity between the two 'V-centered stars', lending further support to the $\mathbf{G a}_{3} \mathbf{V E t}$ formulation. Because of the constraints imposed by the several chelate rings, the $\mathrm{O} 3-\mathrm{V}_{\text {central }}-\mathrm{O} 3$ angles departure significantly from the octahedral figures [values of 76.7(4), 91.0(3), 104.8(5) and 160(4) for $\mathbf{G a}_{3} \mathbf{V E t}$, Tables S2 and S3], resulting in the trigonally distorted coordination sphere, as illustrated in Figure S2. The helical pitch angle, defined as the dihedral angle between the $\mathrm{M}_{4}$ and the $\mathrm{M}_{\text {central }}(\mathrm{O})_{2} \mathrm{M}_{\text {peripheral }}$ planes, is larger in $\mathbf{G a}_{3} \mathbf{V E t}$ than in $\mathrm{Fe}_{3} \mathbf{C r E t}$; this is consistent with "propeller blades" that are more tilted relative to the $C_{3}$ axis, and a greater extent of structural distortion involving the central ion [30]. The pitch angles shown in Table 1 fit the trend to increasing values on going from Cr- to V- and then to Fe-centered stars, $\left[\mathrm{M}_{3} \mathrm{M}^{\prime}\left(\mathrm{L}^{\mathrm{R}}\right)_{2}(\mathrm{dpm})_{6}\right]$, a trend that has been correlated with increasing axial zero-field splitting parameters $|D|$ in this family of tetranuclear complexes [19, 30, 42].

In the unit cell, the triangular molecules are arranged in pairs whose components are related by an inversion center (Figure S6). As a consequence of the high crystal symmetry, although each tetranuclear molecule is chiral ( $\Lambda$ or $\Delta$ enantiomeric forms), the crystals themselves are non-chiral [43, 44]. Additionally, each central $\mathrm{V}^{\mathrm{III}}$ ion of the pair of molecules sits on four crystallographic proper rotation axes, one threefold (main) and three twofold (perpendicular to and related by the threefold symmetry axis) that, in turn, bisect the propeller blades. Symmetry requirements determine the threefold positional disorder of the ethyl groups in the tripodal ligands, and the resulting (disordered) arrangement has higher symmetry than the isolated molecules themselves. 


\subsection{Magnetic Properties}

The temperature dependence of the magnetic susceptibility of $\mathbf{G a}_{\mathbf{3}} \mathbf{V E t}$ is reported in the form of $\chi \mathrm{T}$ vs. T plot in Fig. 2.

At room temperature, the observed $\chi \mathrm{T}$ value is $1.04 \mathrm{~cm}^{3} \mathrm{~K} \mathrm{~mol}^{-1}$, in agreement with the expected value for $\mathrm{S}=1$ with $\mathrm{g}$ close to 2 . However, on cooling, the $\chi \mathrm{T}$ vs. $\mathrm{T}$ product steadily decreases to reach $0.8 \mathrm{~cm}^{3} \mathrm{~K} \mathrm{~mol}^{-1}$ at $40 \mathrm{~K}$. Below this temperature, the $\chi \mathrm{T}$ value drops to $0.35 \mathrm{~cm}^{3} \mathrm{~K} \mathrm{~mol}^{-1}$ at $2 \mathrm{~K}$. It is evident that such behavior cannot be rationalized on the basis of a simple $\mathrm{S}=1$ Spin Hamiltonian:

$$
\widehat{H}=g \beta \widehat{\boldsymbol{S}} \cdot \vec{B}+D \widehat{S}_{z}^{2}+E\left(\widehat{S}_{x}^{2}-\widehat{S}_{y}^{2}\right)
$$

Indeed, this model is unable to reproduce the linear increase of $\chi \mathrm{T}$ in the hightemperature range. This behavior is indicative of a partially quenched orbital contribution to the magnetic moment of $\mathbf{G a}_{3} \mathbf{V E t}$ which, together with spin-orbit coupling (SOC) results in close-lying excited states. The latter are effective in inducing a sizeable temperature independent paramagnetism (TIP) contribution and, thus, a linear behavior of $\chi \mathrm{T}$ for $\mathrm{T}>50 \mathrm{~K}$ [45]. Indeed, in pure octahedral symmetry, vanadium(III) is a ${ }^{3} T_{1 g}$ state (having two electrons in the $t_{2 g} \mathrm{~d}$ orbital set); this can be split by the combined action of SOC and geometrical distortion of the ligand field from purely octahedral symmetry. This can be described using the following Hamiltonian:

$$
\widehat{H}=\sigma \lambda \widehat{\boldsymbol{L}} \cdot \widehat{\boldsymbol{S}}+\sigma^{2} B_{2}^{0}\left(3 \widehat{L}_{z}^{2}-\widehat{\boldsymbol{L}}^{2}\right)+\sigma^{2} B_{2}^{2}\left(\widehat{L}_{x}^{2}-\widehat{L}_{y}^{2}\right)+\mu_{B}\left(\sigma \widehat{\boldsymbol{L}}+g_{e} \widehat{\boldsymbol{S}}\right) \cdot \vec{B}
$$

where $\sigma$ is the combined orbital reduction factor and $\lambda$ is the SOC constant, while $B_{2}^{0}$ and $B_{2}^{2}$ are the axial and rhombic distortion parameters of the ligand field. As largely discussed in the literature $[15,46]$ and anticipated in the Introduction, when $B_{2}^{0}$ is positive, the $T_{1 g}$ orbital state is split in such a way that a singlet orbital ground state is lying lowest, and the ensuing SOC provides an essentially isolated zero-field-split spin triplet state (see Fig. 3). The situation is different when $B_{2}^{0}$ is negative, since this provides an $E$ orbital ground state; while this cannot be mapped on a Spin Hamiltonian, it can be further split by

Fig. 2 Temperature dependence of $\chi \mathrm{T}$ and field dependence of $\mathrm{M}$ (inset plot) of $\mathbf{G a}_{\mathbf{3}} \mathbf{V E t}$ and best fits [28] obtained using Ligand Field Hamiltonian (2) with parameters reported in the text (continuous line). Dashed lines represent best fit [28] obtained using Spin Hamiltonian (1) with parameters reported in the text

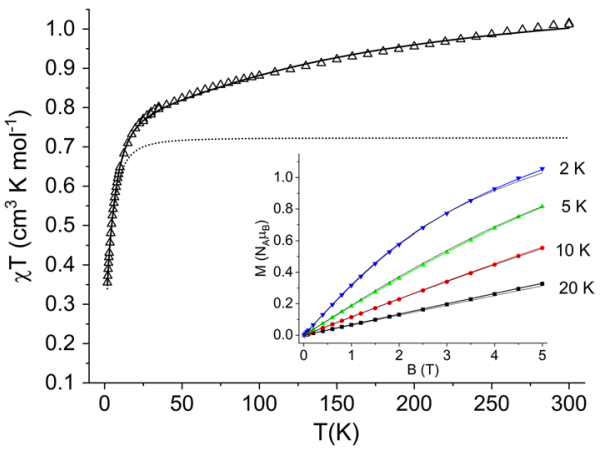




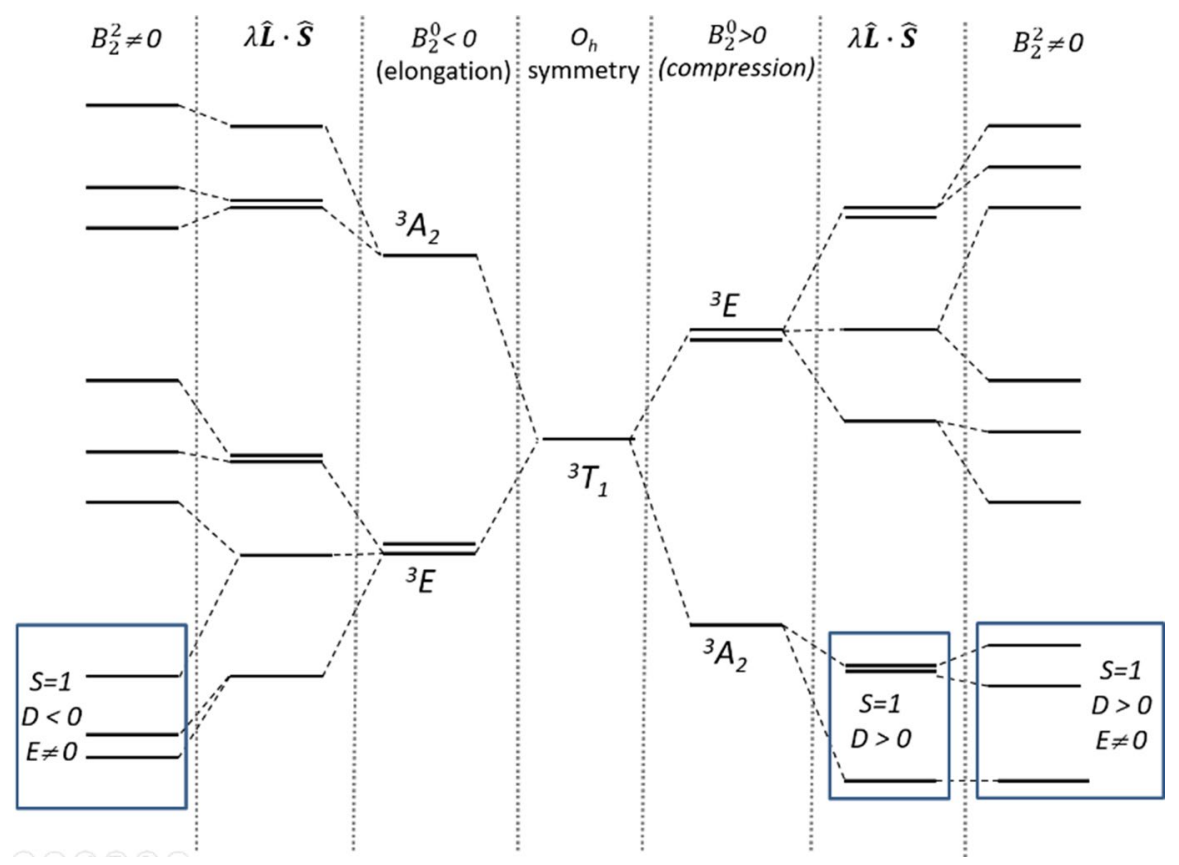

Fig. 3 Scheme of the energy pattern expected for vanadium(III) in distorted octahedral symmetry after application of different perturbations of Hamiltonian (2). Adapted from ref. [9]

rhombic distortion, providing two orbital singlet states, close in energy, each of them with rhombic anisotropy. It is evident, however, that the rhombic distortion is only possible in cases where the point symmetry of the vanadium(III) ion is lower than axial.

Magnetic data, including isothermal magnetization curves, were fitted using (2), as implemented in PHI [28]. The best-fit parameters, which provide a perfect simultaneous reproduction of both $\mathrm{M}$ vs. $\mathrm{H}$ and $\chi \mathrm{T}$ vs. $\mathrm{T}$ curves, were: $B_{2}^{0}$ $=-439 \pm 1 \mathrm{~cm}^{-1}, B_{2}^{2}=148.4 \pm 0.3 \mathrm{~cm}^{-1}$, and $\sigma=-0.734 \pm 0.003$ with $\lambda$ fixed to $75 \mathrm{~cm}^{-1}$. With these values, there is a ground singlet with two close-lying states at 1.67 and $14.97 \mathrm{~cm}^{-1}$; further excited states are at 173.9, 188.2, and $195.8 \mathrm{~cm}^{-1}$, and at 812,813 , and $817 \mathrm{~cm}^{-1}$. It is clear that the levels are now grouped in sets of pseudotriplets: the ground one has essentially easy-axis type, with an effective zero-field splitting of $c a .-14.1 \mathrm{~cm}^{-1}$. Accordingly, Hamiltonian (1) could only be employed to model the low-temperature magnetic properties, i.e., the isothermal magnetization curves, providing as best-fit values $D=-14.1 \pm 0.3 \mathrm{~cm}^{-1}, E=1.21 \pm 0.02 \mathrm{~cm}^{-1}$, and $g=1.72 \pm 0.01$. While the latter value may appear quite low, it is very close to the value derived from HF-EPR of $\mathbf{F e}_{\mathbf{3}} \mathbf{V E t}[9]$. However, this approach is clearly not reproducing the thermal behavior of the magnetic susceptibility above $50 \mathrm{~K}$ (see dotted line in Fig. 3), which is much influenced by the presence of low lying excited states. 


\subsection{HF-EPR Spectroscopy}

HF-EPR spectra, measured at frequencies between 190 and $460 \mathrm{GHz}$ and at $5 \mathrm{~K}$, are reported in Fig. 4. They clearly show a main transition that moves toward zero field on decreasing frequency. No spectrum is observed below $210 \mathrm{GHz}$, indicating a quite large zero-field splitting. A second weaker transition is observed at a somewhat lower field and disappears for frequencies lower than $285 \mathrm{GHz}$. No other identifiable transitions are observed in the investigated field range. Measuring at $5 \mathrm{~K}$ in this frequency range allows getting a high polarization of the spin system with mostly only the lowest energy level populated (the energy of a frequency of $210 \mathrm{GHz}$ corresponds to the one of a $11 \mathrm{~K}$ temperature). Furthermore, the intensity of both transitions decreases when increasing the temperature to $15 \mathrm{~K}$, indicating that they are issued from the lowest energy levels.

By plotting the resonance field of the main transition as a function of frequency, a behavior that is linear at high fields and parabolic at low fields is observed (Fig. 5). This, together with the lineshape, clearly identifies the transition as a perpendicular one, while the resonant field points to a formally forbidden one.

Fig. 4 HF-EPR spectra of a polycrystalline powder sample of $\mathbf{G a}_{3} \mathbf{V E t}$, measured at variable frequencies and $5 \mathrm{~K}$

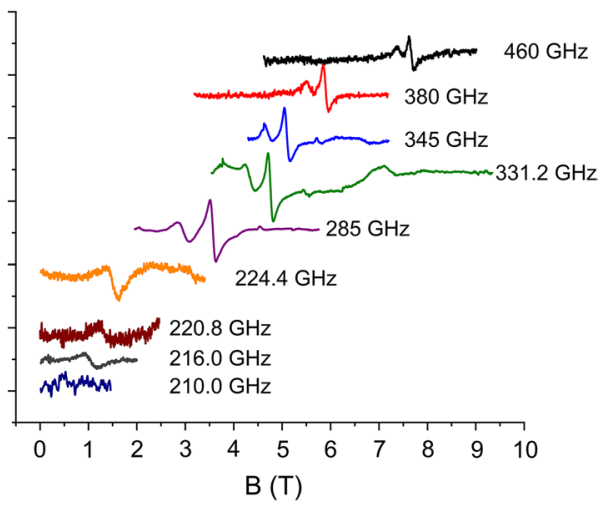

Fig. 5 Frequency dependence of the observed HF-EPR resonant fields of $\mathbf{G a}_{\mathbf{3}} \mathbf{V E t}$ and best fit to the main transition field using expression (3). Black squares are resonant fields of the main transition; empty circles represent the resonant fields of the second transition, observed only for $\mathrm{D} / \mathrm{h} \nu<3 / 4$. See text for details

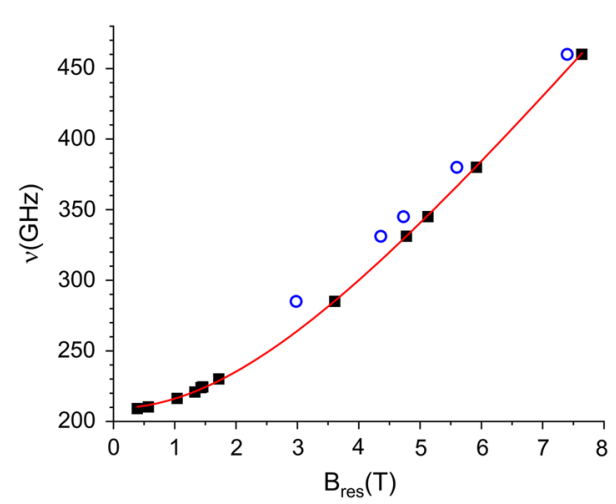


If we assume a Spin Hamiltonian description of our system, the observed transition is the one between the levels labeled as $m_{\mathrm{S}}=\mid 0>$ and $m_{\mathrm{S}}=\mid+1>$ in zero field ( $m_{\mathrm{S}}=\mid+1>$ and $m_{\mathrm{S}}=\mid-1>$ in applied field, thus formally forbidden). We can fit this dependence using the following relation [47]:

$$
\Delta E=\sqrt{\left(4 g_{x, y}^{2} \beta^{2} H^{2}+D^{2}\right)} .
$$

This provides a nice agreement with the experimental data using the following best-fit parameters: $D=209.35 \mathrm{GHz}\left(6.98 \mathrm{~cm}^{-1}\right), g=1.9196$. It is well known [48, 49] that if $D / h \nu<3 / 4$ the forbidden transition at half field can provide two signals in a powder spectrum, one corresponding to a canonical orientation of the field, the other one to an off-axis resonant field.

We then plot in Fig. 6 the angular dependence of the resonant fields for two of the experimentally used frequencies, calculated on the basis of Spin Hamiltonian (1) and the $D$ value obtained by the fit to Eq. (3). This clearly points to the presence of one canonical maximum and one minimum which are observed at $224.4 \mathrm{GHz}$, while one minimum (non-canonical, $\theta=55.5^{\circ}$ ) and two (canonical) maxima are observed at $460 \mathrm{GHz}$. It is, however, worth noting that the $\theta=90^{\circ}$ transition has zero probability for perpendicular polarization of the microwave radiation (i.e., the experimental setup which we used, selecting $\Delta \mathrm{M}_{\mathrm{S}}= \pm 1$ transitions), whereas
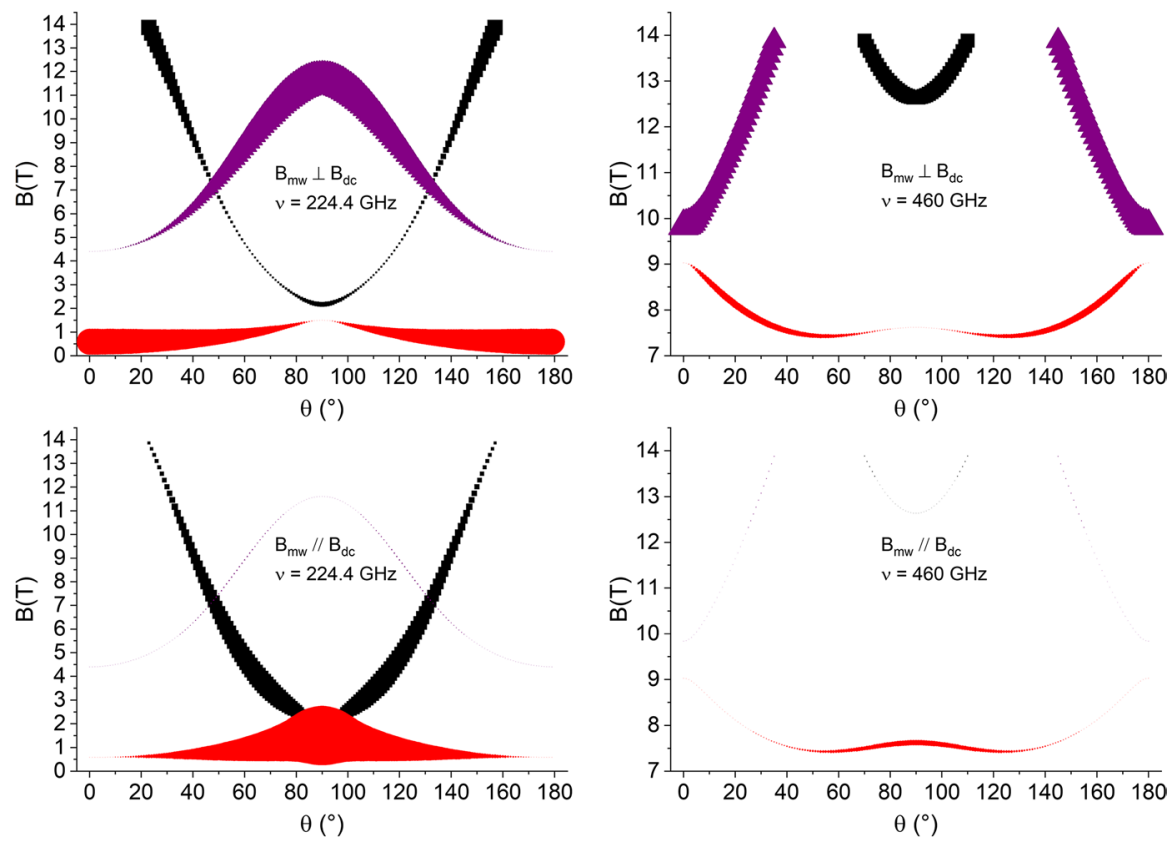

Fig. 6 Angular dependence of the HF-EPR resonance fields of $\mathbf{G a}_{\mathbf{3}} \mathbf{V E t}$ at two frequencies in the $\mathrm{zX}$ plane, using Spin Hamiltonian (1) and parameters obtained by best fit of (3). Size of the symbols is proportional to the transition probability 
its probability is highest for parallel polarization (i.e., with oscillating $\boldsymbol{B}_{\boldsymbol{m} w}$ parallel to $\boldsymbol{B}_{d c}$, selecting $\Delta M_{\mathrm{S}}=0$ transitions). On the contrary, the $\theta=0^{\circ}$ transition has zero probability in parallel polarization and maximum probability for perpendicular polarization (see Fig. 6). By considering the lineshape of the observed transitions and their resonance fields, the observed spectrum should then be assigned to the parallel polarization, which also results in zero probability for the "allowed" transitions. With this approach, reasonable simulations could be obtained for the experimental spectra at different frequencies (see Figure S7). Unfortunately, due to the low sensitivity of the observed "forbidden" transition to the sign of $D$, no conclusion can be reached on this point. Finally, it is worth noting that assuming the same model used to rationalize magnetic data did not lead to reasonable reproduction of the experimental spectra.

\section{Discussion}

The results reported in the previous sections deserve some comments. It is first to be noted that the analysis of magnetic and HF-EPR data provides conflicting indications: for the first set of techniques, the results can only be rationalized by assuming that vanadium(III) in $\mathbf{G a}_{\mathbf{3}} \mathbf{V E t}$ has easy-axis type anisotropy, and its global behavior cannot be traced back to a simple spin triplet, but rather to a ${ }^{3} \mathrm{E}$ ligand field state partially split by rhombic distortion. The presence of a rhombic term in the Hamiltonian is not at odds with the observed trigonal crystallographic symmetry. Indeed, the latter is only a consequence of the statistical disorder of the ethyl pendant on the tris-alkoxo ligand, while the molecular symmetry of $\mathbf{G a} \mathbf{3} \mathbf{V E t}$ is not axial. The results obtained by the magnetic characterization provides a magnitude of single-ion anisotropy for $\mathrm{V}^{\mathrm{III}}$ in the star-shaped compound which would allow rationalizing the global anisotropy of $\mathbf{F e}_{3} \mathbf{V E t}$ without invoking any anisotropic exchange, at variance with what we reported in ref. [9]. Indeed, in the strong exchange approximation, the Zero-Field Splitting tensor of $\mathbf{F e}_{\mathbf{3}} \mathbf{V E t}$ is related to the microscopic anisotropic parameters (single-ion anisotropy tensor and spin-spin interactions) through:

$$
\mathbf{D}_{F e 3 V}=d_{F e}\left(\mathbf{D}_{F e(1)}+\mathbf{D}_{F e(2)}+\mathbf{D}_{F e(3)}\right)+d_{V} \mathbf{D}_{V}+d_{F e-F e} \sum_{i, j=1}^{3(j>i)} \mathbf{D}_{F e(i)-F e(j)}+d_{F e-V} \sum_{i=1}^{3} \mathbf{D}_{F e(i)-V} .
$$

Here, $d_{i}$ 's and $\mathrm{d}_{\mathrm{ij}}$ 's are the projection coefficients on the global anisotropy of spin state $\mathrm{S}$ of the single-ion anisotropies $\left(\mathbf{D}_{\mathrm{Fe}(\mathrm{i})}, \mathbf{D}_{\mathrm{V}}\right)$ and of the spin-spin interaction $\left(\mathbf{D}_{\mathrm{Fe}(\mathrm{i})-\mathrm{Fe}(\mathrm{i})}, \mathbf{D}_{\mathrm{Fe}(\mathrm{i})-\mathrm{V}}\right)$, respectively, which can be calculated recursively [50] (see Table 2). A similar relation hold for the $\mathbf{g}$ tensors:

$$
\mathbf{g}_{F e 3 V}=c_{F e}\left(\mathbf{g}_{F e(1)}+\mathbf{g}_{F e(2)}+\mathbf{g}_{F e(3)}\right)+c_{V} \mathbf{g}_{V} .
$$

It is now firmly established that peripheral $\mathrm{Fe}^{\mathrm{III}}$ ions in star-shaped complexes have hard-axis anisotropy with substantial rhombicity $\left(D_{\mathrm{Fe}} \approx+0.7 \mathrm{~cm}^{-1}, E_{\mathrm{Fe}} \approx\right.$ $0.08 \mathrm{~cm}^{-1}$ ). Furthermore, different HF-EPR and magnetometry studies pointed out that the easy-axis direction of the anisotropy tensor of $\mathrm{Fe}^{\mathrm{III}}$ is approximately 
Table 2 Projection coefficients and calculated dipolar contribution to the anisotropy of the ground state in $\mathrm{Fe}_{3} \mathrm{VEt}$

\begin{tabular}{cccccc}
\hline$d_{F e}$ & $d_{V}$ & $d_{F e-F e}$ & $d_{F e-V}$ & $c_{F e}$ & $c_{V}$ \\
\hline$\frac{17}{140}$ & $\frac{1}{120}$ & $\frac{17}{112}$ & $-\frac{17}{360}$ & $\frac{17}{45}$ & $-\frac{2}{15}$ \\
\hline
\end{tabular}

perpendicular to the plane of the star and that the local intermediate axis is oriented along the Fe-M direction (which is often a symmetry imposed direction [8, 51-53]). If one substitutes the values and orientations expected for $\mathrm{Fe}^{\mathrm{IIII}}$, the dipolar contribution calculated in the point dipole approximation and $\mathbf{D}_{\mathrm{v}}=-14.1 \mathrm{~cm}^{-1}$, which models the splitting in the low-energy region for vanadium(III), the estimated anisotropy for the ground $\mathrm{S}=13 / 2$ state of $\mathbf{F e}_{3} \mathbf{V E t}$ from Eq. (4) is $-0.290 \mathrm{~cm}^{-1}$, in good agreement with the experimentally determined value in ref [9] $\left(D\left(\mathrm{Fe}_{3} \mathrm{~V}^{E t}\right)=\right.$ $\left.-0.314 \mathrm{~cm}^{-1}\right)$. It is further to be noted that the experimentally determined [9] $g$ value for $\mathbf{F e}_{3} \mathbf{V E t}$ requires $g_{V}=1.7$ which is exactly the value one obtains by modeling the low-temperature magnetic data of $\mathbf{G a}_{3} \mathbf{V E t}$ with a ground pseudotriplet (see above).

On the other hand, HF-EPR data can be rationalized assuming a standard spin triplet Spin Hamiltonian, resulting from a positive value of the trigonal distortion of the ligand field, and, thus, in an orbital singlet ground state. This would confirm our previous hypothesis, based essentially on the literature data $[15,16,46]$, of positive zero-field splitting for vanadium(III) in the $\mathbf{F e}_{3} \mathbf{V E t}$ cluster. It is, however, to be noted that appropriate simulation of the HF-EPR spectra required to assume, as discussed, parallel polarization of the microwave radiation. This is not the experimental setup used, thus raising further issues about these data. Similar problems with the radiation polarization were recently reported for an experimental study of a Hobased polyoxometalate (POM) single-molecule magnet [54].

\section{Conclusions}

We have reported a preliminary characterization of a $\mathbf{G a}_{\mathbf{3}} \mathbf{V E t}$ cluster both from a structural, magnetic, and spectroscopic point of view. The observed results do not allow to obtain a firm conclusion as to the anisotropy of central vanadium(III) in $\mathbf{G a}_{3} \mathbf{V E t}$ and, thus, in the isostructural SMM $\mathrm{Fe}_{3} \mathbf{V E t}$. Indeed, while the magnetic measurements point to an easy-axis type anisotropy with a relevant rhombicity, HF-EPR spectra can only be interpreted on the assumption of an easy-plane type anisotropy, of smaller magnitude but with some rhombicity. Also, given the small amount of sample available for HF-EPR measurements, some weak signals, associated with the allowed transitions and giving more stringent constraints on the anisotropy parameters, may have been missed. This confirms the difficulties in getting a reliable characterization of vanadium(III)-containing complexes. Despite that we have succeeded in stabilizing it with tripodal ligand and in exploiting its anisotropy in $\mathbf{F e}_{3} \mathbf{V E t}$ with a triplication of magnetic blocking temperature compared to the 
archetypal $\mathrm{Fe}_{4} \mathrm{SMM}$, a rational design of optimized SMMs based on this metal ion is still challenging. In perspective, it might be useful to extend the HF-EPR study to higher fields and frequencies using a larger amount of sample to obtain more sound conclusions. This would allow to obtain clearer indication as to the contribution of the anisotropic exchange to the anisotropy of this system. In this perspective single crystal techniques, such as cantilever torque magnetometry, which proved very powerful in the investigation of the lanthanide-based variant of tetranuclear star-shaped SMM [55], might also prove useful.

Acknowledgements We are grateful to Dr. André Luis Rüdiger for collecting the single-crystal X-ray diffraction data for $\mathbf{G a}_{3} \mathbf{V E t}$.

Authors' Contributions LS and RS designed the study. All authors performed the experiments. LS and JFS wrote the manuscript with input from all authors.

Funding Open access funding provided by Università degli Studi di Firenze within the CRUI-CARE Agreement. This study was financially supported by Fundação Araucária (grant 283/2014, protocol 37509), Conselho Nacional de Desenvolvimento Científico e Tecnológico (CNPq, grant 308426/20169), and Coordenação de Aperfeiçoamento de Pessoal de Nível Superior (CAPES, Finance Code 001). K.C.M.W., F.S.S., D.L.H., and J.F.S. thank the Brazilian agencies CNPq and CAPES for fellowships. R.S. and L.S. acknowledge the funding of Fondazione Cassa Risparmio Firenze and MIUR ("Progetto Dipartimenti di Eccellenza 2018-2022”).

\section{Compliance with Ethical Standards}

Conflict of interest The authors declare that there is no conflict of interest.

Electronic Supplementary Material Crystallographic data for $\mathbf{G a}_{3} \mathbf{V E t}$ have been deposited with the Cambridge Crystallographic Data Centre (CCDC, https://www.ccdc.cam.ac.uk). Deposition number: 2008356. Online Resource contains crystal and structure refinement data, further crystallographic figures, infrared spectroscopy characterization and further analysis of HF-EPR data.

Open Access This article is licensed under a Creative Commons Attribution 4.0 International License, which permits use, sharing, adaptation, distribution and reproduction in any medium or format, as long as you give appropriate credit to the original author(s) and the source, provide a link to the Creative Commons licence, and indicate if changes were made. The images or other third party material in this article are included in the article's Creative Commons licence, unless indicated otherwise in a credit line to the material. If material is not included in the article's Creative Commons licence and your intended use is not permitted by statutory regulation or exceeds the permitted use, you will need to obtain permission directly from the copyright holder. To view a copy of this licence, visit http://creativecommons.org/licen ses/by/4.0/.

\section{References}

1. R. Sessoli, D. Gatteschi, A. Caneschi, M.A. Novak, Nature 365, 141 (1993)

2. D. Gatteschi, R. Sessoli, J. Villain, Molecular Nanomagnets (Oxford University Press, Oxford, 2007)

3. A. Caneschi, D. Gatteschi, R. Sessoli, A.L. Barra, L.C. Brunel, M. Guillot, J. Am. Chem. Soc. 113, 5873 (1991)

4. T. Glaser, Chem. Commun. 47, 116 (2011) 
5. G. Aromí, E.K. Brechin, Struct. Bond. 122, 1 (2006)

6. M. Fittipaldi, L. Sorace, A.-L. Barra, C. Sangregorio, R. Sessoli, D. Gatteschi, Phys. Chem. Chem. Phys. 11, 6555 (2009)

7. A.-L. Barra, A. Caneschi, A. Cornia, F.F. de Biani, D. Gatteschi, C. Sangregorio, R. Sessoli, L. Sorace, F. Fabrizi de Biani, D. Gatteschi, C. Sangregorio, R. Sessoli, L. Sorace, J. Am. Chem. Soc. 121, 5302 (1999)

8. E. Tancini, M.J. Rodriguez-Douton, L. Sorace, A.-L. Barra, R. Sessoli, A. Cornia, Chem. Eur. J. 16, $10482(2010)$

9. K.C.M. Westrup, M.-E. Boulon, P. Totaro, G.G. Nunes, D.F. Back, A. Barison, M. Jackson, C. Paulsen, D. Gatteschi, L. Sorace, A. Cornia, J.F. Soares, R. Sessoli, Chem. A Eur. J. 20, 13681 (2014)

10. A. Cornia, M. Mannini, R. Sessoli, D. Gatteschi, Eur. J. Inorg. Chem. 2019, 552 (2019)

11. A. Cornia, M. Mannini, P. Sainctavit, R. Sessoli, Chem. Soc. Rev. 40, 3076 (2011)

12. D. Gatteschi, A. Cornia, M. Mannini, R. Sessoli, Inorg. Chem. 48, 3408 (2009)

13. G. Serrano, L. Poggini, M. Briganti, A.L. Sorrentino, G. Cucinotta, L. Malavolti, B. Cortigiani, E. Otero, P. Sainctavit, S. Loth, F. Parenti, A.-L. Barra, A. Vindigni, A. Cornia, F. Totti, M. Mannini, R. Sessoli, Nat. Mater. 19, 546 (2020)

14. P.L.W. Tregenna-Piggott, S.P. Best, H.U. Gudel, H. Weihe, C.C. Wilson, J. Solid State Chem. 145, $460(1999)$

15. P.L.W. Tregenna-Piggott, G. Carver, Inorg. Chem. 43, 8061 (2004)

16. K.R. Kittilstved, L.A. Sorgho, N. Amstutz, P.L.W. Tregenna-Piggott, A. Hauser, Inorg. Chem. 48, 7750 (2009)

17. I.S. Tidmarsh, L.J. Batchelor, E. Scales, R.H. Laye, L. Sorace, A. Caneschi, J. Schnack, E.J.L. McInnes, Dalton Trans. 43, 9402 (2009)

18. D.D. Perrin, W.L. Armarego, Purification of Laboratory Chemicals (Butterworth-Heinemann, Oxford, 1997)

19. P. Totaro, K.C.M. Westrup, M.-E. Boulon, G.G. Nunes, D.F. Back, A. Barison, S. Ciattini, M. Mannini, L. Sorace, J.F. Soares, A. Cornia, R. Sessoli, Dalton Trans. 42, 4416-4426 (2013)

20. L.E. Manzer, Inorg. Synth. 21, 135-140 (1982)

21. Bruker APEX2 software. Bruker AXS Inc., Madison, Wisconsin, USA (2010)

22. G.M. Sheldrick, Acta Crystallogr. Sect. A Found. Crystallogr. 64, 112 (2008)

23. G.M. Sheldrick, Acta Crystallogr. Sect. C Struct. Chem. 71, 3 (2015)

24. A.J.C. Wilson (ed.), International Tables for X-Ray Crystallography (Kluwer Academic Publishing, Dordrecht, 1992)

25. L.J. Farrugia, J. Appl. Crystallogr. 32, 837 (1999)

26. L.J. Farrugia, J. Appl. Crystallogr. 45, 849 (2012)

27. G.A. Bain, J.F. Berry, J. Chem. Educ. 85, 532 (2008)

28. N.F. Chilton, R.P. Anderson, L.D. Turner, A. Soncini, K.S. Murray, J. Comput. Chem. 34, 1164 (2013)

29. S. Stoll, A. Schweiger, J. Magn. Reson. 178, 42 (2006)

30. S. Accorsi, A.L. Barra, A. Caneschi, G. Chastanet, A. Cornia, A.C. Fabretti, D. Gatteschi, C. Mortalo, E. Olivieri, F. Parenti, P. Rosa, R. Sessoli, L. Sorace, W. Wernsdorfer, L. Zobbi, J. Am. Chem. Soc. 128, 4742 (2006)

31. H. Putz, K. Brandenburg, DIAMOND - Crystal and Molecular Structure Visualization software. Crystal Impact GbR, Bonn, Germany (2006)

32. M.J. Rodriguez-Douton, M. Mannini, L. Armelao, A.L. Barra, E. Tancini, R. Sessoli, A. Cornia, Chem. Commun. 47, 1467 (2011)

33. R. van Eldick, Inorganic High Pressure Chemistry: Kinetics and Mechanisms (Elsevier, Amsterdam, 1986)

34. C.H. Langford, H.B. Gray, Ligand Substitution Processes (W. A. Benjamin, New York, 1965)

35. K. Saito, H. Kido, A. Nagasawa, Coord. Chem. Rev. 100, 427 (1990)

36. A.D. Hugi, L. Helm, A.E. Merbach, Helv. Chim. Acta 68, 508 (1985)

37. R.B. Jordan, Reaction Mechanisms of Inorganic and Organometallic Systems, 2nd edn. (Oxford University Press, New York, 1998)

38. R.G. Pearson, J. Chem. Educ. 45, 581 (1968)

39. P.W. Ayers, R.G. Parr, R.G. Pearson, J. Chem. Phys. 124, 194107 (2006)

40. R.D. Shannon, C.T. Prewitt, Acta Crystallogr. Sect. B Struct. Crystallogr. Cryst. Chem. 25, 925 (1969) 
41. R.D. Shannon, Acta Crystallogr. Sect. A 32, 751 (1976)

42. L. Gregoli, C. Danieli, A.-L. Barra, P. Neugebauer, G. Pellegrino, G. Poneti, R. Sessoli, A. Cornia, Chem. A Eur. J. 15, 6456 (2009)

43. H.D. Flack, Helv. Chim. Acta 86, 905 (2003)

44. H.D. Flack, G. Bernardinelli, Chirality 20, 681 (2008)

45. K.R. Dunbar, E.J. Schelter, A.V. Palii, S.M. Ostrovsky, V.Y. Mirovitskii, J.M. Hudson, M.A. Omary, S.I. Klokishner, B.S. Tsukerblat, J. Phys. Chem. A 107, 11102 (2003)

46. P.L.W. Tregenna-Piggott, H. Weihe, J. Bendix, A.-L. Barra, H.-U. Güdel, Inorg. Chem. 38, 5928 (1999)

47. F.E. Mabbs, D. Collison, Electron Paramagnetic Resonance of $d$ Transition Metal Compounds (Elsevier, Amsterdam, 1998)

48. J.H. van der Waals, M.S. de Groot, Mol. Phys. 2, 333 (1959)

49. J. Reedijk, B. Nieuwenhuijse, Recl. Des Trav. Chim. Des Pays-Bas 91, 533 (2010)

50. A. Bencini, D. Gatteschi, EPR of Exchange-Coupled Systems (Springer, Berlin, 1990)

51. L. Vergnani, A.-L. Barra, P. Neugebauer, M.J. Rodriguez-Douton, R. Sessoli, L. Sorace, W. Wernsdorfer, A. Cornia, Chem. A Eur. J. 18, 3390 (2012)

52. L. Sorace, M.-E. Boulon, P. Totaro, A. Cornia, J. Fernandes-Soares, R. Sessoli, Phys. Rev. B Condens. Matter Mater. Phys. 88, 104407 (2013)

53. L. Rigamonti, A. Cornia, A. Nava, M. Perfetti, M.-E. Boulon, A.-L. Barra, X. Zhong, K. Park, R. Sessoli, Phys. Chem. Chem. Phys. 16, 17220 (2014)

54. S. Ghosh, S. Datta, L. Friend, S. Cardona-Serra, A. Gaita-Ariño, E. Coronado, S. Hill, Dalton Trans. 41, 13697 (2012)

55. L. Rigamonti, A. Nava, M.-E. Boulon, J. Luzon, R. Sessoli, A. Cornia, Chem. A Eur. J. 21, 12171 (2015)

Publisher's Note Springer Nature remains neutral with regard to jurisdictional claims in published maps and institutional affiliations. 